\title{
Enabling Dense Spatial Reuse in mmWave Networks
}

\author{
Suraj Jog, Jiaming Wang, Haitham Hassanieh, Romit Roy Choudhury \\ University of Illinois at Urbana Champaign
}

\begin{abstract}
Millimeter Wave (mmWave) networks can deliver multiGbps wireless links that use extremely narrow directional beams. This provides us with a new way to exploit spatial reuse in order to scale network throughput. In this work, we present MilliNet, the first millimeter wave network that can exploit dense spatial reuse to allow many links to operate in parallel in a confined space and scale the wireless throughput with the number of clients. Results from a $60 \mathrm{GHz}$ testbed show that MilliNet can deliver a total wireless network data rate of more than $38 \mathrm{Gbps}$ for 10 clients which is $5.8 \times$ higher than current 802.11 mmWave standards.
\end{abstract}

\section{CCS CONCEPTS}

- Networks $\rightarrow$ Network protocols; Wireless access networks;

\section{KEYWORDS}

Millimeter Wave, Spatial Reuse, VR, Beam Alignment

\section{INTRODUCTION}

Millimeter wave (mmWave) technology promises to revolutionize wireless LANs by delivering multi-Gbps wireless links [3-5]. It also differs from other wireless technologies in that it uses very directional steerable narrow beams. As a result, the traditional interference model on which we had built 802.11 wireless networks no longer holds. Consider for example the current broadcast model shown in Fig. 1(a). Whenever a node is transmitting, all other nodes must stay silent to avoid interference since the entire medium is shared. In contrast, the use of very narrow beams in mmWave networks allows several APs and clients to transmit and receive simultaneously on the same channel without interfering, as shown in Fig. 1(b). Hence, mmWave presents a significant opportunity for exploiting extremely dense spatial reuse to enable many links to simultaneously communicate at multiGbps data rates. This would enable exciting new applications

Permission to make digital or hard copies of all or part of this work for personal or classroom use is granted without fee provided that copies are not made or distributed for profit or commercial advantage and that copies bear this notice and the full citation on the first page. Copyrights for components of this work owned by others than ACM must be honored. Abstracting with credit is permitted. To copy otherwise, or republish, to post on servers or to redistribute to lists, requires prior specific permission and/or a fee. Request permissions from permissions@acm.org.

SIGCOMM Posters '18, August 20-25, 2018, Budapest, Hungary

(c) 2018 Association for Computing Machinery.

ACM ISBN 978-1-4503-5915-3/18/08...\$15.00

https://doi.org/10.1145/3234200.3234241

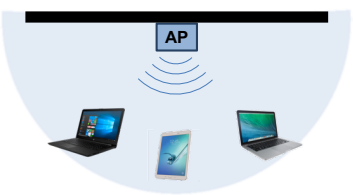

(a) Traditional Wireless LAN

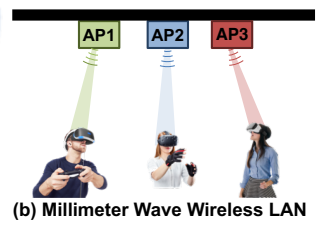

Figure 1: Spatial reuse in mmWave vs traditional WiFi networks.

like multi-user wireless virtual reality for education and training where high bandwidth data must be streamed to each user in real-time [1, 2]. It will also enable robotic factory automation where robots stream continuous real-time video back to control servers [6].

This paper presents MilliNet, the first millimeter wave network that enables many densely packed APs and clients to simultaneously communicate without interfering. Enabling extremely dense spatial reuse in MilliNet, however, requires addressing two questions:

(1) How do we efficiently align the beams of the APs and clients in 3D space in a manner that avoids interference and maximizes the number of links that can operate simultaneously? Due to the directional nature of communication, the beams of APs and clients need to be aligned towards each other prior to data transmission. However, in a network with multiple links (AP-client pairs), simply aligning the beams of each link along the Line-of-sight (direct) path can end up creating more interference due to multipath reflections that severly impacts some clients. Instead, MilliNet aligns the beams along both the direct and reflected paths in order to maximize the number of links that can operate concurrently. However, to be fair among clients, MilliNet generates several such alignments and runs a three stage scheduling algorithm to schedule transmissions of different links along different paths, while ensuring that each client gets its fair share to communicate at its maximum achievable data rate.

(2) How do we adapt the beams in realtime to accommodate mobility and changes in the environments? Even a single client movement can affect the interference between all the other links in a network. Hence, to find the new best scheduling, we must remeasure the interference and rediscover all paths between APs and clients which would require collecting $O\left(N^{2}\right)$ measurements for a network with $N$ APs and $N$ clients. This would result in a prohibitively high overhead which cannot scale and would prevent us from adapting to changes in the environment and supporting mobile clients. This is why past mmWave work that can support multiple links is designed for static networks with predictable interference models [7].

Instead, MilliNet exploits the beam tracking mechanism for mobile clients, and designs a mmWave protocol that folds 

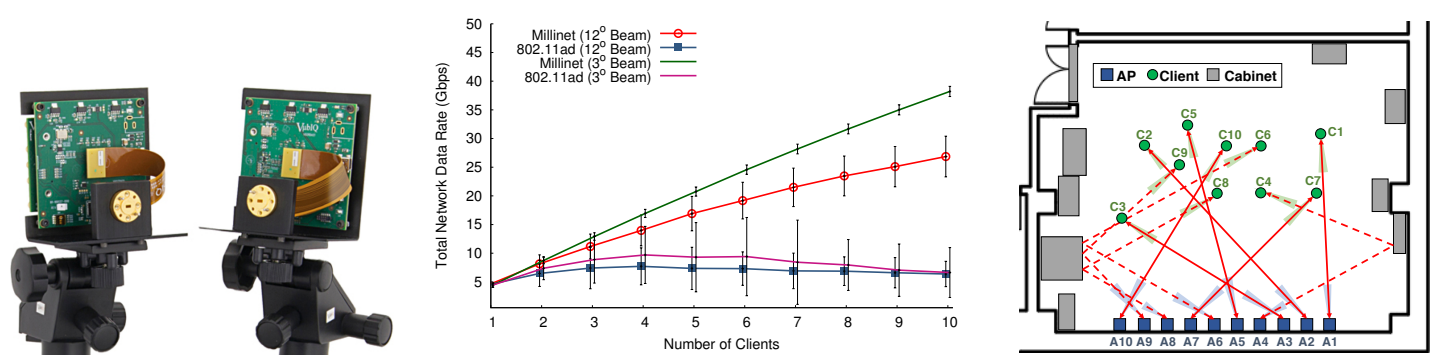

Figure 2: (left) $60 \mathrm{GHz}$ radios used in evaluation. (middle) Total network data rate versus number of clients. (right) Example of MilliNet's beam selection in practice.

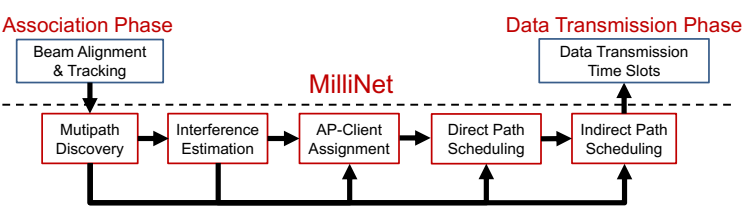

Figure 3: Overview of MilliNet's System Architecture.

multipath discovery and interference detection into the beam alignment and tracking. MilliNet then coordinates APs to share measurements over the Ethernet allowing it to discover all multipath and interference without incurring additional overhead. Hence, MilliNet continuously maintains an up-todate view of the multipath and interference in the network as clients move allowing to adapt and self-reconfigure to achieve the best performance.

We have designed MilliNet to be backward compatible with the current mmWave wireless LAN standard 802.11ad/ay making it easy to integrate into future standards. We have implemented MilliNet using $60 \mathrm{GHz}$ wireless radios and demonstrated its ability to enable dense spatial reuse.

\section{MILLINET SYSTEM ARCHITECTURE}

MilliNet maintains the same high level structure as 802.11ad, which divides time into beacon intervals. Each beacon interval has two phases: Association Phase where clients associate with APs and align their beams, and the Data Transmission phase. MilliNet's architectural flow is shown in Fig. 3. It uses a controller that sits between the Association Phase and the Data Transmission phase of the protocol. During the Association Phase, MilliNet collects path and interference information and runs its scheduling algorithm which dictates the AP-client scheduling in the data transmission phase.

MilliNet starts with an Association Phase similar to 802.11ad, where the APs and clients sweep their beams to collect information about the directions in which their signals can reach other APs and clients. This information is then fed to the MilliNet controller. The controller first discovers all the paths connecting any two nodes in the network and then uses beam pattern models to estimate the interference created between links by aligning the beams along each path. MilliNet uses these results to find the best beam configuration that maximizes the number of AP-client pairs that can communicate simultaneously. To reduce the complexity of the system, MilliNet solves this problem in three stages:

- Stage 1: Assign each client to communicate with one AP for the duration of the entire beacon interval. This avoids the need for fast hand-off within the beacon interval.

- Stage 2: Schedule the maximal set of AP-client pairs that can communicate along their direct or highest-throughput path without interfering with each other.

- Stage 3: Schedule additional links along alternate reflected paths in order to maximize throughput, such that the links coexist with each other and with the direct path links scheduled in Stage 2.

The above results in a TDMA schedule of transmissions between APs and clients along different paths during each time slot of the data transmission phase. The entire process is repeated every beacon interval to adapt to changes in the environment and accommodate client mobility.

\section{IMPLEMENTATION \& RESULTS}

We have implemented MilliNet by using extensive measurements of multipath and interference from an indoor $60 \mathrm{GHz}$ wireless testbed equipped with directional antennas of beamwidth equal to $12^{\circ}$ and $3^{\circ}$. We ran trace driven simulations using ns 3 and verified our results by empirically testing that any two pairs of APs and clients can transmit without interfering. Fig. 2 shows the radios used in our implementation as well as the results.

For a testbed with 10 APs and clients packed in an area of 860 sq.ft., MilliNet can scale the overall network data rate with the number of clients delivering over $38 \mathrm{Gbps}$ for 10 clients (Fig. 2). Furthermore, compared to the current 802.11ad standard's, MilliNet can increase the per client throughput by $4.2 \times$ for $12^{\circ}$ beamwidth and $5.8 \times$ for $3^{\circ}$. Finally, Fig. 2 also shows an example snapshot of a time slot where MilliNet aligns the beams in a manner that enables all 10 APs and clients to communicate at the same time without interfering, hence, demonstrating MilliNet's ability to enable extreme spatial reuse. 


\section{REFERENCES}

[1] Omid Abari, Dinesh Bharadia, Austin Duffield, and Dina Katabi. 2017. Enabling High-Quality Untethered Virtual Reality, In NSDI. 14th USENIX Symposium on Networked Systems Design and Implementation (NSDI 17) (2017).

[2] Adam Connor-Simons. 2016. Enabling wireless virtual reality. (2016).

[3] IEEE Standards Association. 2012. IEEE Standards 802.11ad-2012: Enhancements for Very High Throughput in the $60 \mathrm{GHz}$ Band. (2012).

[4] Markets and Markets. [n. d.]. Millimeter Wave Technology Market worth 4,632.8 Million USD by 2022 . ([n. d.]).

[5] Thomas Nitsche, Carlos Cordeiro, Adriana B Flores, Edward W Knightly, Eldad Perahia, and Joerg C Widmer. 2014. IEEE 802.11 ad: directional $60 \mathrm{GHz}$ communication for multi-Gigabit-per-second Wi-Fi. IEEE Communications Magazine 52, 12 (2014), 132-141.

[6] Victoria Turk. 2016. These Supermarket Warehouse Robots Have Their Own Mobile Network. (2016).

[7] X. Zhou, Z. Zhang, Y. Zhu, Y. Li, S. Kumar, A. Vahdat, B. Y. Zhao, and H Zheng. 2012. Mirror Mirror on the Ceiling: Flexible Wireless Links for Data Centers. In ACM SIGCOMM. 